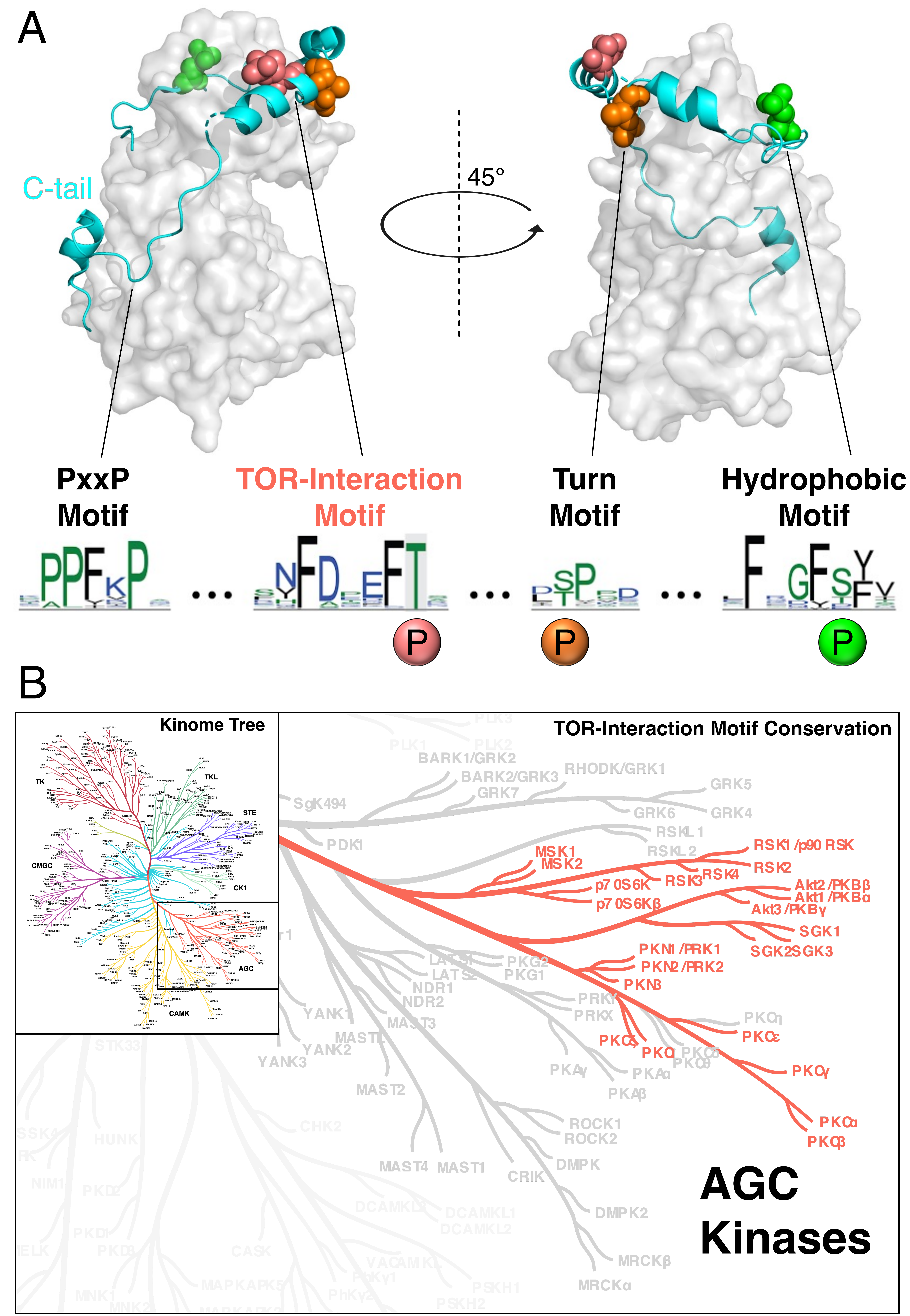

Figure 1 


\section{mTOR Regulation of AGC Kinases: New Twist to an Old Tail}

Timothy R. Baffi and Alexandra C. Newton ${ }^{*}$

Department of Pharmacology, University of California at San Diego, La Jolla, CA 92093, USA

*Correspondence to: anewton@health.ucsd.edu

Running title: Regulation of AGC kinases by mTORC2

Text pages: 16

Figures: 2

References: 45

Abstract: 233 words

Abbreviations: mechanistic target of rapamycin, mTOR; phosphoinositide-dependent kinase 1, PDK1; protein kinase A, PKA; protein kinase B, PKB; protein kinase C, PKC 


\section{Abstract:}

The family of AGC kinases not only regulate cellular biology by phosphorylating substrates, but are themselves controlled by phosphorylation. Phosphorylation generally occurs at two conserved regions in these kinases: a loop near the entrance to the active site, termed the activation loop, that correctly aligns residues for catalysis, and a C-terminal tail whose phosphorylation at a site termed the hydrophobic motif stabilizes the active conformation. Whereas phosphorylation of the activation loop is well established to be catalyzed by the phosphoinositide-dependent kinase 1 (PDK1), the mechanism of phosphorylation of the C-tail hydrophobic motif has been controversial. For a subset of AGC kinases, which includes most protein kinase $\mathrm{C}(\mathrm{PKC})$ isozymes and Akt, phosphorylation of the hydrophobic motif in cells was shown to depend on mTORC2 over 15 years ago, yet whether by direct phosphorylation or by another mechanism has remained elusive. The recent identification of a novel and evolutionarily conserved phosphorylation site on the C-tail termed the TOR-Interaction Motif (TIM) has finally unraveled the mystery of how mTORC2 regulates its client kinases. mTORC2 does not directly phosphorylate the hydrophobic motif, rather it converts kinases such as PKC and Akt into a conformation that can ultimately autophosphorylate at the hydrophobic motif. Identification of the direct mTOR phosphorylation that facilitates auto-regulation of the C-tail hydrophobic motif revises the activation mechanisms of mTOR-regulated AGC kinases. This new twist to an old tail opens avenues for therapeutic intervention.

Significance Statement: The enzyme mTORC2 has been an enigmatic regulator of AGC kinases such as protein kinase $\mathrm{C}$ (PKC) and Akt. The recent discovery of a motif named the TOR 
Interaction Motif in the C-tail of these kinases solves the mystery: mTORC2 marks these kinases for maturity by, ultimately, facilitating autophosphorylation another C-tail site, the hydrophobic motif.

\section{The Activation Loop: A Protein Kinase Activation Switch}

A common feature of nearly all protein kinases involves the structuring of a flexible activation loop in the kinase domain, which predominantly occurs by phosphorylation (Adams, 2001; Nolen et al., 2004). This phosphorylation event converts the kinase into an active conformation, which enables phosphorylation of substrates (Nolen et al., 2004; Taylor et al., 2012, 2019). The activation loop site of distinct kinase subfamilies is often regulated by a master activation loop kinase, as is the case for AMPK-related kinases phosphorylated by LKB1 (Lizcano et al., 2004), CDK kinases phosphorylated by CDK7 (Harper and Elledge, 1998), and AGC kinases, which are phosphorylated by the PDK1 (Mora et al., 2004). AGC family kinases, named after member protein kinases A, G, and C, are distinguished by a conserved C-terminal extension of the kinase domain (C-tail) (Figure 1A) (Pearce et al., 2010), which serves as both an intrinsic modulator of catalytic activity and a regulatory handle for interacting proteins (Kannan et al., 2007). PDK1 binds to the C-tail of AGC kinases including protein kinase A (PKA) (Cheng et al., 1998), protein kinase B (PKB/Akt) (Alessi et al., 1997; Stokoe et al., 1997), and PKC (Dutil et al.) to phosphorylate and activate these kinases. PDK1 is capable of autophosphorylation at its own 
activation loop (Wick et al., 2003), and its activity is regulated through recognition of substrates (Toker and Newton, 2000a). Thus, for AGC kinases the C-tail is the critical feature linking the recruitment of the upstream kinase PDK1 to the phosphorylation of its activation loop and subsequent kinase activation.

\section{The Search for the Hydrophobic Motif Kinase "PDK2"}

The AGC kinase C-tail itself is additionally regulated by phosphorylation, harboring two highly conserved sites, the turn motif (Keranen et al., 1995) and the hydrophobic motif (Edwards and Newton, 1997; Pearson et al., 1995). Turn motif phosphorylation has an established role in stabilizing the kinase domain (Keshwani et al., 2012), whereas the hydrophobic motif enhances catalytic activity. The identification of PDK1 as the activation loop kinase (Mora et al., 2004) galvanized a decade-long search for the elusive hydrophobic motif kinase "PDK2" (Chan and Tsichlis, 2001). Several kinases, including MAPKAP-K2 and the mechanistic target of rapamycin (mTOR) kinase complex mTORC1, DNA-PK, ATM,ILK, p38 MAPK, and NEK6 emerged as hydrophobic motif kinase candidates (Dong and Liu, 2005); however, these findings were not able to be replicated in both an in vitro and cellular context.

In 2005, a distinct, rapamycin-insensitive mTOR complex, mTORC2 (Sarbassov et al., 2004), was shown to be required for hydrophobic motif phosphorylation and catalytic activity of Akt (Sarbassov et al., 2005) and, subsequently, numerous other AGC kinase family members, including six of the nine PKC isozymes $(\alpha, \beta, \gamma, \varepsilon, \zeta, \imath)$ (Facchinetti et al., 2008; Ikenoue et al., 2008) and SGK (Garcia-Martinez and Alessi, 2008) family kinases. S6-kinase family members are 
alternatively dependent upon mTORC1 for phosphorylation of their hydrophobic motif (Guertin et al., 2006; Isotani et al., 1999) due to a conserved TOR-signaling motif that confers mTORC1 specificity (Ali and Sabatini, 2005; Schalm and Blenis, 2002). mTORC2 has been shown to directly phosphorylate the turn motif site co-translationally on Akt (Oh et al., 2010) and PKC (Tobias et al., 2015); however, the mechanism for regulation of the hydrophobic motif has been hotly debated. Although mTORC2 was proposed to regulate the hydrophobic motif by direct phosphorylation, mTORC2 could not effectively phosphorylate the hydrophobic motif site of PKC in vitro (Ikenoue et al., 2008). Furthermore, phosphomimetics at the C-tail sites in PKC, although functionally tolerated, do not bypass the requirement for mTORC2 (Baffi et al., 2021a). mTORC2 has been reported to be capable of increasing Akt hydrophobic motif phosphorylation and activity in vitro (Sarbassov et al., 2005); however, purified Akt exhibits constitutive turn motif phosphorylation, confounding mTORC2 regulation of both of these sites (Ikenoue et al., 2008; Sarbassov et al., 2005). What is more, pure PKC and Akt have been shown to effectively autophosphorylate at the hydrophobic motif in vitro, and their catalytic activity is necessary for phosphorylation at this site in cells, suggesting that mTORC2 is not the direct hydrophobic motif kinase (Behn-Krappa and Newton, 1999; Sarbassov et al., 2005; Toker and Newton, 2000b). Thus, the mechanism by which mTORC2 regulates hydrophobic motif phosphorylation and the activity of AGC kinases, remained unclear.

\section{mTOR Regulation of AGC Kinases Revealed}


The recent identification of a novel and conserved C-tail phosphorylation site termed the TOR interaction motif (TIM) provides the long-awaited mechanism for PKC and Akt activation by mTORC2 (Baffi et al., 2021a). The TIM precedes the well-characterized turn motif by seven residues and comprises a highly conserved $\mathrm{F}-\mathrm{x}_{3}-\mathrm{F}-\mathrm{pT}$ motif in the C-tail (Figure $1 \mathrm{~A}$ ). Found in all 5 taxonomical groups throughout evolution, the TIM is conserved in the six PKC isozymes that require mTORC2, as well as mTOR-dependent Akt, S6K, SGK, PKN, and RSK family kinases (Figure 1B). Whereas many mTOR-independent AGC kinases harbor the turn motif phosphorylation site, the TIM is conserved exclusively in mTOR-dependent AGC kinases. Notably, this motif is absent in AGC kinases such as PKA that are not regulated by mTOR, suggesting that the TIM phosphorylation is the critical site specifying regulation by mTOR. TIM phosphorylation, either alone or in combination with turn motif phosphorylation, is necessary for kinase activity: Ala substitution at this site in Akt, and at both this site and the turn motif in PKC, were shown to abolish kinase activity in live-cell assays, recapitulating the effects of mTORC2 deletion or inhibition (Baffi et al., 2021a). These mutations prevented phosphorylation of the activation and hydrophobic motif sites, mirroring the effect of mTORC2 absence.

Biochemical studies further revealed that the mTORC2 step is effectively bypassed by overexpression of PDK1 (Baffi et al., 2021a). In cells lacking functional mTORC2, Akt and mTORdependent PKC isozymes are not significantly phosphorylated at any of the C-tail sites. Restoration of functional mTORC2 permits phosphorylation at both the turn motif and the hydrophobic motif, resulting in active kinases. But active kinase can also be obtained by overexpressing PDK1: this kinase promotes phosphate incorporation at its bona fide site, the activation loop, and also at the hydrophobic motif, but not at the bona fide mTORC2 site, the 
turn motif. Importantly, the PDK1 rescue depends on the intrinsic catalytic activity of both PDK1 and the client kinase, as kinase-dead mutants of PKC or Akt could not rescue hydrophobic motif phosphorylation upon PDK1 overexpression (Baffi et al., 2021a). Thus, these findings suggest that mTORC2 regulates hydrophobic motif autophosphorylation by facilitating PDK1 phosphorylation of the activation loop. Therefore, TIM phosphorylation by mTORC2 marks AGC kinases for activation by PDK1.

\section{PKC: A Case Study in TIM Phosphorylation}

Extensive mechanistic studies on how C-tail phosphorylation regulates PKC allowed it to serve as a good model AGC kinase to dissect the role of TIM phosphorylation (Baffi et al., 2021a). PKC is constitutively phosphorylated following translation to yield a catalyticallycompetent and stable enzyme with a half-time of approximately 30 minutes (Borner et al., 1989; Keranen et al., 1995). Despite the duration of these processing steps, PKC phosphorylations are so tightly coupled that they cannot be temporally distinguished experimentally. Inhibition or genetic ablation of mTORC2 slows the rate of PKC processing, demonstrating that $\mathrm{mTORC2}$ regulates the first and rate-limiting step of the maturation process, which culminates in phosphorylation of the hydrophobic motif (Baffi et al., 2021a). This finding raises the question: How does TIM phosphorylation facilitate PKC maturation?

Analysis of a reported structure of the kinase domain of $P K C \beta I I$ revealed a previously unappreciated symmetrical homodimer (Grodsky et al., 2006), the interface of which is coordinated, strikingly, by helices containing the TOR-Interaction Motif (Figure 2A). Because 
mature (phosphorylated) PKC is well established to be monomeric, the observation of a dimer interface of the isolated kinase domain led to the idea that newly-synthesized PKC may dimerize, with mTORC2 phosphorylation of the TIM disrupting the dimer interface to facilitate the transition to the monomeric, phosphorylated, and catalytically-competent species that engages in signaling. This hypothesis proposes that TIM phosphorylation by mTORC2 facilitates binding of PDK1 to monomerized PKC by exposing the C-tail, initiating the conformational switches leading to autophosphorylation at the hydrophobic motif. Unphosphorylated PKC is thermally unstable (Bornancin and Parker, 1996, 1997; Edwards and Newton, 1997) and rapidly degraded in the cell (Baffi et al., 2019; Van et al., 2021); thus, dimerization may aide in stabilizing/protecting nascent PKC during processing. Biophysical assays showed that phosphorylation of the TIM and turn motif sites dissociates the PKC dimer, initiating the cascade of processing phosphorylations that yields a catalytically-competent kinase (Baffi et al., 2021a). Therefore, the role of TIM phosphorylation during PKC maturation is to expose the Ctail for PDK1 to bind, leading to activation loop phosphorylation and hydrophobic motif cisautophosphorylation. As a consequence of hydrophobic motif phosphorylation, PKC isozymes adopt an autoinhibited conformation in which its activity is restrained by an autoinhibitory pseudosubstrate segment (Baffi et al., 2019). Autoinhibited PKC isozymes are catalytically competent and stable, poised to respond to second messengers that initiate downstream signaling at cellular membranes (Figure 2B).

\section{mTORC2 converts kinases into an autophosphorylation-competent mode}


The regulation of eukaryotic protein kinases by autophosphorylation is a prevalent mechanism in kinase biology (Beenstock et al., 2016), particularly well studied for receptor tyrosine kinases, where ligand-induced dimerization promotes autophosphorylation (Bae and Schlessinger, 2010; Jura et al., 2011). Many kinases catalyze their own autophosphorylation on their activation loop to convert into an active conformation, and this ability to autophosphorylate generally depends on a priming event that renders the kinase "prone-to-autophosphorylate" (Beenstock et al., 2016). This can be induced by ligand binding, protein:protein interactions, or priming phosphorylations. The findings reviewed above reveal that a large branch of the AGC group of kinases, defined by the presence of the TIM (Figure 1), may be converted into a "prone-toautophosphorylate" state by mTORC2 (Figure 2B). This work opens avenues to understand how members other than PKC and Akt are regulated by mTORC2-facilitated autophosphorylation.

\section{Therapeutic Implications for Novel AGC Activation Mechanisms}

Uncovering the mTORC2 mechanism has important ramifications for the use of mTOR inhibitors in the clinic for cancer: these inhibitors will prevent the phosphorylation of PKC and cause its loss, compromising the utility of these drugs (Sun, 2021). However, elucidation of the function of mTORC2 in relieving PKC dimerization unveils a potential approach to desensitize PKC to mTOR inhibitors. Specifically, dimer disruptors (such as stapled peptides (Baffi et al., 2021a)) that mimic this function of mTORC2 could be used in combination with mTOR inhibitors. These dimer disruptors would dissociate the nascent PKC dimer to promote the binding of PDK-1, thus initiating the maturation of PKC to the fully-phosphorylated, 
autoinhibited, and stable species. Such an approach is predicted to increase the effectiveness of mTOR inhibitors.

\section{Conclusion}

The aforementioned study clarifies the direct kinases of the conserved phosphorylation sites in PKC and Akt, which may be applicable to the other mTOR-regulated AGC kinases that harbor these sites. mTORC2 directly phosphorylates the turn motif and TIM sites, a priming step, facilitating PDK1 phosphorylation of the activation loop and intramolecular autophosphorylation of the hydrophobic motif that activates the kinase (Figure 2C). New insights into the phosphorylation mechanisms of protein kinases expand our understanding of the requirements for kinase activation and potentiate new therapeutic strategies. For example, we recently proposed modulating PKC levels by targeting the PHLPP phosphatase family that regulates its dephosphorylation and degradation (Baffi et al., 2021b; Tovell and Newton, 2021). Alternatively, as noted above, disrupting the TIM dimer interface could be used to increase the levels of phosphorylated and stable PKC in diseases where PKC activity is diminished, as is the case in cancer (Antal et al., 2015; Newton and Brognard, 2017). Whereas TIM phosphorylation promotes PDK1 phosphorylation of the activation loop for PKC and Akt, this site may play additional roles in other kinases, which merits further investigation for these kinases and the pathophysiological signaling they mediate in a variety of diseases. Thus, identification of the TIM phosphorylation provides the long-awaited mechanism for mTORC2 regulation of the hydrophobic motif and reveals an unappreciated dimerization step in PKC maturation amenable to targeted therapy. 
Authorship contribution:

Wrote or contributed to the writing of the manuscript, Baffi, T. R., and Newton, A. C. 


\section{References:}

Adams, J.A. (2001). Kinetic and catalytic mechanisms of protein kinases. Chem. Rev. 101, 22712290.

Alessi, D.R., James, S.R., Downes, C.P., Holmes, A.B., Gaffney, P.R.J., Reese, C.B., and Cohen, P. (1997). Characterization of a 3-phosphoinositide-dependent protein kinase which phosphorylates and activates protein kinase Ba. Curr. Biol. 7, 261-269.

Ali, S.M., and Sabatini, D.M. (2005). Structure of S6 kinase 1 determines whether raptor-mTOR or rictor-mTOR phosphorylates its hydrophobic motif site. J. Biol. Chem. 280, 19445-19448. Antal, C.E., Hudson, A.M., Kang, E., Zanca, C., Wirth, C., Stephenson, N.L., Trotter, E.W., Gallegos, L.L., Miller, C.J., Furnari, F.B., et al. (2015). Cancer-associated protein kinase C mutations reveal kinase's role as tumor suppressor. Cell 160, 489-502.

Bae, J.H., and Schlessinger, J. (2010). Asymmetric tyrosine kinase arrangements in activation or autophosphorylation of receptor tyrosine kinases. Mol. Cells 29.

Baffi, T.R., Van, A.-A.N., Zhao, W., Mills, G.B., and Newton, A.C. (2019). Protein Kinase C Quality Control by Phosphatase PHLPP1 Unveils Loss-of-Function Mechanism in Cancer. Mol. Cell 74, 378-392.e5.

Baffi, T.R., Lordén, G., Wozniak, J.M., Feichtner, A., Yeung, W., Kornev, A.P., King, C.C., Del Rio, J.C., Limaye, A.J., Bogomolovas, J., et al. (2021a). mTORC2 controls the activity of PKC and Akt by phosphorylating a conserved TOR interaction motif. Sci. Signal. 14, eabe4509.

Baffi, T.R., Katsenelson, K.C., and Newton, A.C. (2021b). PHLPPing the Script: Emerging Roles of PHLPP Phosphatases in Cell Signaling. Annu. Rev. Pharmacol. Toxicol. 61, annurev-pharmtox031820-122108. 
Beenstock, J., Mooshayef, N., and Engelberg, D. (2016). How Do Protein Kinases Take a Selfie (Autophosphorylate)? Trends Biochem. Sci. 41, 938-953.

Behn-Krappa, A., and Newton, A.C. (1999). The hydrophobic phosphorylation motif of conventional protein kinase $\mathrm{C}$ is regulated by autophosphorylation. Curr. Biol. 9, 728-737. Bornancin, F., and Parker, P.J. (1996). Phosphorylation of threonine 638 critically controls the dephosphorylation and inactivation of protein kinase Ca. Curr. Biol. 6, 1114-1123.

Bornancin, F., and Parker, P.J. (1997). Phosphorylation of protein kinase C-alpha on serine 657 controls the accumulation of active enzyme and contributes to its phosphatase-resistant state.

J. Biol. Chem. 272, 3544-3549.

Borner, C., Filipuzzi, I., Wartmann, M., Eppenberger, U., and Fabbro, D. (1989). Biosynthesis and posttranslational modifications of protein kinase C in human breast cancer cells. J Biol Chem $264,13902-13909$.

Chan, T.O., and Tsichlis, P.N. (2001). PDK2: A Complex Tail in One Akt. Sci. Signal. 2001, pe1pe1.

Cheng, X., Ma, Y., Moore, M., Hemmings, B.A., and Taylor, S.S. (1998). Phosphorylation and activation of cAMP-dependent protein kinase by phosphoinositide-dependent protein kinase. Proc. Natl. Acad. Sci. U. S. A. 95, 9849-9854.

Dong, L.Q., and Liu, F. (2005). PDK2: The missing piece in the receptor tyrosine kinase signaling pathway puzzle. Am. J. Physiol. - Endocrinol. Metab. 289.

Dutil, E.M., Toker, A., and Newton, A.C. Regulation of conventional protein kinase C isozymes by phosphoinositide-dependent kinase 1 (PDK-1). Curr. Biol. 8, 1366-1375.

Edwards, A.S., and Newton, A.C. (1997). Phosphorylation at conserved carboxyl-terminal 
hydrophobic motif regulates the catalytic and regulatory domains of protein kinase C. J. Biol. Chem. 272, 18382-18390.

Facchinetti, V., Ouyang, W., Wei, H., Soto, N., Lazorchak, A., Gould, C., Lowry, C., Newton, A.C., Mao, Y., Miao, R.Q., et al. (2008). The mammalian target of rapamycin complex 2 controls folding and stability of Akt and protein kinase C. EMBO J. 27, 1932-1943.

Garcia-Martinez, J.M., and Alessi, D.R. (2008). mTOR complex 2 (mTORC2) controls hydrophobic motif phosphorylation and activation of serum- and glucocorticoid-induced protein kinase 1 (SGK1). Biochem J 416, 375-385.

Grodsky, N., Li, Y., Bouzida, D., Love, R., Jensen, J., Nodes, B., Nonomiya, J., and Grant, S. (2006). Structure of the catalytic domain of human protein kinase $\mathrm{C}$ beta II complexed with a bisindolylmaleimide inhibitor. Biochemistry 45, 13970-13981.

Guertin, D.A., Stevens, D.M., Thoreen, C.C., Burds, A.A., Kalaany, N.Y., Moffat, J., Brown, M., Fitzgerald, K.J., and Sabatini, D.M. (2006). Ablation in mice of the mTORC components raptor, rictor, or mLST8 reveals that mTORC2 is required for signaling to Akt-FOXO and PKCalpha, but not S6K1. Dev Cell 11, 859-871.

Harper, J.W., and Elledge, S.J. (1998). The role of Cdk7 in CAK function, a retro-retrospective. Genes Dev. 12, 285-289.

Ikenoue, T., Inoki, K., Yang, Q., Zhou, X., and Guan, K.-L. (2008). Essential function of TORC2 in PKC and Akt turn motif phosphorylation, maturation and signalling. EMBO J. 27, 1919-1931. Isotani, S., Hara, K., Tokunaga, C., Inoue, H., Avruch, J., and Yonezawa, K. (1999). Immunopurified mammalian target of rapamycin phosphorylates and activates p70 S6 kinase a in vitro. J. Biol. Chem. 274, 34493-34498. 
Jura, N., Zhang, X., Endres, N.F., Seeliger, M.A., Schindler, T., and Kuriyan, J. (2011). Catalytic Control in the EGF Receptor and Its Connection to General Kinase Regulatory Mechanisms. Mol. Cell 42.

Kannan, N., Haste, N., Taylor, S.S., and Neuwald, A.F. (2007). The hallmark of AGC kinase functional divergence is its C-terminal tail, a cis-acting regulatory module. Proc. Natl. Acad. Sci. U. S. A. $104,1272-1277$.

Keranen, L.M., Dutil, E.M., and Newton, A.C. (1995). Protein kinase C is regulated in vivo by three functionally distinct phosphorylations. Curr. Biol. 5, 1394-1403.

Keshwani, M.M., Klammt, C., von Daake, S., Ma, Y., Kornev, A.P., Choe, S., Insel, P.A., and Taylor, S.S. (2012). Cotranslational cis-phosphorylation of the COOH-terminal tail is a key priming step in the maturation of cAMP-dependent protein kinase. Proc Natl Acad Sci U S A 109, E1221-9.

Lizcano, J.M., Göransson, O., Toth, R., Deak, M., Morrice, N.A., Boudeau, J., Hawley, S.A., Udd, L., Mäkelä, T.P., Hardie, D.G., et al. (2004). LKB1 is a master kinase that activates 13 kinases of the AMPK subfamily, including MARK/PAR-1. EMBO J. 23, 833-843.

Metz, K.S., Deoudes, E.M., Berginski, M.E., Jimenez-Ruiz, I., Aksoy, B.A., Hammerbacher, J., Gomez, S.M., and Phanstiel, D.H. (2018). Coral: Clear and Customizable Visualization of Human Kinome Data. Cell Syst. 7, 347-350.e1.

Mora, A., Komander, D., van Aalten, D.M.F., and Alessi, D.R. (2004). PDK1, the master regulator of AGC kinase signal transduction. Semin. Cell Dev. Biol. 15, 161-170.

Newton, A.C., and Brognard, J. (2017). Reversing the Paradigm: Protein Kinase C as a Tumor Suppressor. Trends Pharmacol Sci 38, 438-447. 
Nolen, B., Taylor, S., and Ghosh, G. (2004). Regulation of Protein Kinases: Controlling Activity through Activation Segment Conformation. Mol. Cell 15, 661-675.

Oh, W.J., Wu, C., Kim, S.J., Facchinetti, V., Julien, L.-A., Finlan, M., Roux, P.P., Su, B., and Jacinto, E. (2010). mTORC2 can associate with ribosomes to promote cotranslational phosphorylation and stability of nascent Akt polypeptide. EMBO J. 29, 3939-3951.

Pearce, L.R., Komander, D., and Alessi, D.R. (2010). The nuts and bolts of AGC protein kinases. Nat. Rev. Mol. Cell Biol. 11.

Pearson, R.B., Dennis, P.B., Han, J.W., Williamson, N.A., Kozma, S.C., Wettenhall, R.E., and Thomas, G. (1995). The principal target of rapamycin-induced p70s6k inactivation is a novel phosphorylation site within a conserved hydrophobic domain. EMBO J. 14, 5279-5287. Sarbassov, D.D., Ali, S.M., Kim, D.H., Guertin, D.A., Latek, R.R., Erdjument-Bromage, H., Tempst, P., and Sabatini, D.M. (2004). Rictor, a novel binding partner of mTOR, defines a rapamycininsensitive and raptor-independent pathway that regulates the cytoskeleton. Curr Biol 14, 1296-1302.

Sarbassov, D.D., Guertin, D.A., Ali, S.M., and Sabatini, D.M. (2005). Phosphorylation and regulation of Akt/PKB by the rictor-mTOR complex. Science 307, 1098-1101.

Schalm, S.S., and Blenis, J. (2002). Identification of a conserved motif required for mTOR signaling. Curr. Biol. 12, 632-639.

Stokoe, D., Stephens, L.R., Copeland, T., Gaffney, P.R.J., Reese, C.B., Painter, G.F., Holmes, A.B., McCormick, F., and Hawkins, P.T. (1997). Dual Role of Phosphatidylinositol-3,4,5-trisphosphate in the Activation of Protein Kinase B. Science (80-. ). 277, 567-570.

Sun, S.Y. (2021). mTOR-targeted cancer therapy: great target but disappointing clinical 
outcomes, why? Front. Med. 15, 221-231.

Taylor, S.S., Keshwani, M.M., Steichen, J.M., and Kornev, A.P. (2012). Evolution of the eukaryotic protein kinases as dynamic molecular switches. Philos. Trans. R. Soc. B Biol. Sci. 367, 2517-2528.

Taylor, S.S., Meharena, H.S., and Kornev, A.P. (2019). Evolution of a dynamic molecular switch. IUBMB Life 71, 672-684.

Tobias, I.S., Kaulich, M., Kim, P.K., Simon, N., Jacinto, E., Dowdy, S.F., King, C.C., and Newton, A.C. (2015). Protein kinase $C \zeta$ exhibits constitutive phosphorylation and phosphatidylinositol3,4,5-triphosphate-independent regulation. Biochem. J. BJ20151013.

Toker, A., and Newton, A.C. (2000a). Cellular signaling: Pivoting around PDK-1. Cell 103, 185188.

Toker, A., and Newton, A.C. (2000b). Akt/protein kinase B is regulated by autophosphorylation at the hypothetical PDK-2 site. J. Biol. Chem. 275, 8271-8274.

Tovell, H., and Newton, A.C. (2021). PHLPPing the balance: restoration of protein kinase C in cancer. Biochem. J. 478, 341-355.

Van, A.-A.N., Kunkel, M.T., Baffi, T.R., Lordén, G., Antal, C.E., Banerjee, S., and Newton, A.C. (2021). Protein kinase C fusion proteins are paradoxically loss of function in cancer. J. Biol. Chem. 296, 100445.

Wick, M.J., Ramos, F.J., Chen, H., Quon, M.J., Dong, L.Q., and Liu, F. (2003). Mouse 3Phosphoinositide-dependent Protein Kinase-1 Undergoes Dimerization and transPhosphorylation in the Activation Loop. J. Biol. Chem. 278, 42913-42919. 
Footnotes:

This work was supported by the National Institute of Health General Medicine [Grant R35

GM122523]; T.R.B. was supported by the PhRMA Foundation Pre Doctoral Fellowship in Pharmacology Toxicology [Grant \#20183844] and the UCSD Graduate Training Program in Cellular and Molecular Pharmacology [T32 GM007752].

Financial disclosures: none 
Figure Captions:

Figure 1. TIM is a Conserved Phosphorylation Site in mTOR-Dependent AGC Kinases. A) Crystal structure of the isolated kinase domain of Protein Kinase $C \beta I I(2 \mathrm{IOE})$ showing the conserved Cterminal tail (C-tail; turquoise). The C-tail phosphorylation sites are indicated: TIM (pink; Thr634 in PKC $\beta$ II), turn motif (orange; Thr641 in PKC $\beta I I$ ), and hydrophobic motif (green; Ser660 in PKC $\beta$ II). Sequence logos display conserved features of the AGC C-tail for all mammalian kinases containing the TIM Thr (F-x3-F-pT), including a conserved PXXP motif (Kannan et al., 2007). B) AGC branch of the Human Kinome Tree highlighting in red kinases with the TOR-Interaction Motif (Metz et al., 2018). Adapted from (Baffi et al., 2021a). Reprinted with permission from AAAS.

Figure 2. TIM Phosphorylation Controls PKC Dimerization and Stability. A) Crystal structure of the Protein Kinase $C \beta I I$ kinase domain (2IOE) dimer showing dimerization interface coordinated by the TIM helix. B) Schematic for PKC processing by phosphorylation. Newly-synthesized PKC exists as an inactive dimer (UNPRIMED). Shortly after synthesis, mTORC2 binds and phosphorylates the TIM and turn motif sites at the dimer interface, dissociating the dimer and exposing the C-tail for binding of PDK1. Phosphorylation of the activation loop by PDK1 correctly aligns residues for catalysis, leading to intramolecular autophosphorylation at the hydrophobic motif. The catalytically-competent PKC species then adopts autoinhibited conformation that is stable and poised to become activated by second-messengers (PRIMED). C) Diagram summarizing upstream kinases for PKC and Akt phosphorylations: mTOR catalyzes the phosphorylation of the TIM and turn motif, PDK1 phosphorylates the activation loop, and 
autophosphorylation modifies the hydrophobic motif. This phosphorylation pattern may be applicable to other mTOR-dependent AGC kinases. Adapted from (Baffi et al., 2021a). Reprinted with permission from AAAS. 
